\title{
Aportes de una metodología lúdica al aprendizaje de los estudiantes que cursan la cátedra de Morfofisiología en la Facultad de Cultura Física, Deporte y Recreación*
}

\author{
Melisa Andrea Narváez Duarte** \\ Juan Pablo Macías Nova** \\ Duvan Felipe Acosta Corredor ${ }^{* *+*}$ \\ Francisco Javier Fonseca Zamora**** \\ Lady Johanna Ruíz González $z^{* * * * * *}$
}

Recibido: mayo 3 de 2018 • Aceptado: agosto 15 de 2018

\begin{abstract}
"Artículo de investigación, con financiación propia, vinculado al proyecto de investigación Fodeín Una observación praxiológica de las experiencias de ocio y recreación al interior de la Facultad de Cultura Física, Deporte y Recreación, desarrollado por el grupo de investigación Cuerpo, sujeto y educación, línea Manifestaciones Sociales de Ocio y la recreación (MASOR). Citar como: Narváez, M., Macías, J., Acosta, D., Fonseca, F. y Ruíz, L. (2018). Aportes de una metodología lúdica al aprendizaje de los estudiantes que cursan la cátedra de Morfofisiología en la Facultad de Cultura Física, Deporte y Recreación. Cuerpo, Cultura y Movimiento, 8(2), 63-82. DOI: https://doi.org/10.15332/2422474X.5127
\end{abstract}

"* Estudiante de pregrado de la Facultad de Cultura Física, Deporte y Recreación, X semestre. Universidad Santo Tomás, Bogotá D. C., Colombia. Correo electrónico: melisanarvaez@usantotomas.edu.co ORCID: https://orcid.org/0000-0002-9531-9422

"**"Estudiante de pregrado de la Facultad de Cultura Física, Deporte y Recreación, X semestre. Universidad Santo Tomás, Bogotá D. C., Colombia. Correo electrónico: juan.macias@ usantotomas.edu.co / ORCID: https://orcid.org/0000-0002-5420-5576

"*** Estudiante de pregrado de la Facultad de Cultura Física, Deporte y Recreación, X semestre. Universidad Santo Tomás, Bogotá D. C., Colombia. Correo electrónico: duvanacosta@ usantotomas.edu.co

"***** Docente de la Facultad de Cultura Física, Deporte y Recreación de la Universidad Santo Tomás, Bogotá D. C., Colombia. Investigador de la línea de investigación Manifestaciones Sociales del Ocio y la Recreación (MASOR). Grupo de investigación Cuerpo Sujeto y Educación. Correo electrónico: franciscofonseca@usantotomas.edu.co / ORCID: https://orcid. org/0000-0002-0106-6680

"Docente de la Facultad de Cultura Física, Deporte y Recreación de la Universidad Santo Tomás, Bogotá D. C., Colombia. Investigador de la línea de investigación Manifestaciones Sociales del Ocio y la Recreación (MASOR). Grupo de investigación Cuerpo Sujeto y Educación. Correo electrónico: ladyruizg@usantotomas.edu.co / ORCID: https://orcid. org/0000-0003-3189-4089 


\section{Resumen}

El proyecto se enfocó en establecer los aportes derivados de una metodología lúdica en el aprendizaje de los estudiantes que cursan la cátedra de Morfofisiología en la Facultad de Cultura Física, Deporte y Recreación de la Universidad Santo Tomás, sede Bogotá. La investigación contó con un enfoque mixto: de tipo explicativo y microetnográfico, con una muestra poblacional compuesta por 24 estudiantes pertenecientes al curso de Morfofisiología 2D 2018-1. En la fase cuantitativa se pretendía un incremento en las calificaciones de los estudiantes en esta cátedra, sin embargo este no fue significativo para el estudio; mientras que en la fase cualitativa se identificaron aportes relacionados con la importancia de un ambiente motivador y que generara felicidad en el aprendizaje de los estudiantes, facilitando con este la cohesión grupal y los procesos asociativos por medio del juego. Como conclusión se obtuvo que la combinación entre la educación tradicional y la lúdica es potenciadora en el proceso de enseñanza-aprendizaje..

Palabras clave: aprendizaje, didáctica, juego, lúdica, morfofisiología. 


\section{Contributions of a ludic methodology to the learning of the students of Morphophysiology in the Faculty of Physical Culture, Sports and Recreation}

Abstract

The project focused on establishing the contributions derived from a playful methodology in the learning of the students who study the Morphophysiology Chair in the Faculty of Physical Culture, Sport and Recreation of the Santo Tomás University, Bogotá Headquarters. The research had a mixed approach: of explanatory and microethnographic type, with a population sample composed of 24 students belonging to the course of 2D Morphophysiology 2018-1. In the quantitative phase an increase in the grades of the students in this chair was intended, but this was not significant for the study; while in the qualitative phase, contributions related to the importance of a motivating environment and that generated happiness in students' learning were identified, facilitating group cohesion and associative processes through play. As a conclusion it was obtained that the combination between traditional and playful education is an enhancer in the teaching-learning process.

Keywords: learning, playful, game, didactic, morphophysiology. 


\section{Introducción}

La pedagogía juega un papel importante en el desarrollo de aprendizajes del ser humano, es por esto que en la escuela se debe promover el gusto por el conocimiento, tal como lo menciona Saver (1997) citado por Calzadilla (2004):

La pedagogía se ocupa en su esencia del conocimiento, en el tiempo y en el espacio, de las acciones imprescindibles y necesarias que han de realizarse para que tales procesos resulten, a la postre, eficientes y eficaces, tanto para el educando como para el educador. (p. 124)

Siguiendo lo postulado por Torres y Girón (2009), todo proceso educativo requiere una teoría y una práctica, según esto, la importancia de fomentar un ambiente lúdico en los espacios institucionales de educación superior, hace parte del proceso de desarrollo integral de los estudiantes, pues aunque estas entidades tengan un enfoque académico principalmente, los ambientes lúdicos contribuyen a fortalecer y desarrollar diversas habilidades blandas en los estudiantes, formando un profesional con el conocimiento suficiente y las características humanas que lo distinguen como un ser integral y parte esencial para el desarrollo de la sociedad. De acuerdo con lo anterior, cuando se menciona el concepto "lúdico", se está haciendo referencia al proceso de desarrollo que abarca las diversas dimensiones del ser humano, cuya principal fuente de expresión es el juego (Jiménez, 1998, citado por Posada, 2014; Anzola, Castellanos, Orduz, Salazar y Sanz, 2013).

Con el fin de desarrollar un ambiente lúdico es importante que los docentes se apropien de conceptos relacionados con la "metodología lúdica" la cual esta descrita por Zuluaga y Gómez (2016) como una herramienta para que el estudiante construya su propio conocimiento, relacionando las estructuras mentales con las que cuenta y las que elabora mediante las vivencias.

Una de las cátedras fundamentales en temas de salud que es dictada en la Facultad de Cultura Física, Deporte y Recreación, es la de Morfofisiología, la cual cuenta con una alta tasa de pérdida dentro de la Facultad de acuerdo con la información brindada por el Departamento de Registro y Control de la Universidad. Dentro de los antecedentes revisados, se observa que aspectos como la lúdica y el juego generan grandes aportes al proceso 
de aprendizaje; en cuanto al juego este exige al docente un cambio radical de su papel en el aula: debe dejar de ser un observador del proceso y pasar a ser un transmisor de conocimientos (Sarlé, 2001). Además, el juego es entendido por Piaget citado por Anzola et al. (2013) como un elemento fundamental para potencializar la inteligencia, abarcando aspectos como la lógica y la razón, permitiendo al individuo mejorar su adaptación hacia el contexto. Entendiendo esto, el juego es una herramienta clave y versátil que puede utilizar el maestro para potencializar la inteligencia de sus estudiantes. Por lo tanto, esta cátedra se toma como una oportunidad para aplicar una metodología lúdica frente a una metodología basada principalmente en el aprendizaje memorístico, buscando resaltar aspectos positivos para los actores de la institución al igual que la mejora en el rendimiento académico por parte de los estudiantes.

Es importante en esta cátedra, al ser fundamental en el desarrollo formativo de los estudiantes, sobre todo en el área de la salud, estimular el aprendizaje significativo, descrito por Ausubel (1976) y Moreira (1997) citado por Rodríguez (2004) el cual se logra cuando los individuos identifican los aspectos cognitivos más relevantes de una temática, denominada por el autor como "subsumidores" o "ideas de anclaje", las cuales permiten relacionar los conocimientos adquiridos con los esquemas cognitivos estructurados anteriormente; de esta manera si se logra estimular el aprendizaje significativo en este cátedra que es una de la primeras por cursar en el área de la salud, seguramente las ideas de anclaje y los esquemas de conocimiento ayudarán a los estudiantes a recordar de una manera más eficiente aprendizajes que recibieron anteriormente.

Debido a que el enfoque metodológico de la investigación es de carácter mixto, se cuenta con la hipótesis de que existe una diferencia significativa entre la media de calificaciones del grupo control y la media de calificaciones del grupo experimental, mientras que las categorías a priori relacionadas se basan en las apreciaciones de los estudiantes frente a la metodología de aprendizaje (AFM), las manifestaciones emocionales de la metodología vivenciada (MM) y los aspectos preliminares para la intervención (API). 


\section{Justificación}

La presente investigación se encuentra vinculada al grupo Cuerpo, Sujeto y Educación, perteneciente a la línea emergente de investigación Manifestaciones Sociales del Ocio y la Recreación (MASOR), cuyo principal objetivo es indagar las manifestaciones y conceptualizaciones de la recreación como encargo social. Esta investigación contribuye al fortalecimiento de los recursos producidos por el semillero de investigación, donde se pretende mostrar los efectos de la recreación, en este caso por medio de las prácticas recreativas en la realidad educativa. En la Figura 1 se presenta una de las evidencias de la implementación del material didáctico en el grupo experimental.

Figura 1. Organización de pequeños grupos y entrega de material didáctico. Fotografía Melisa Narváez, 2018

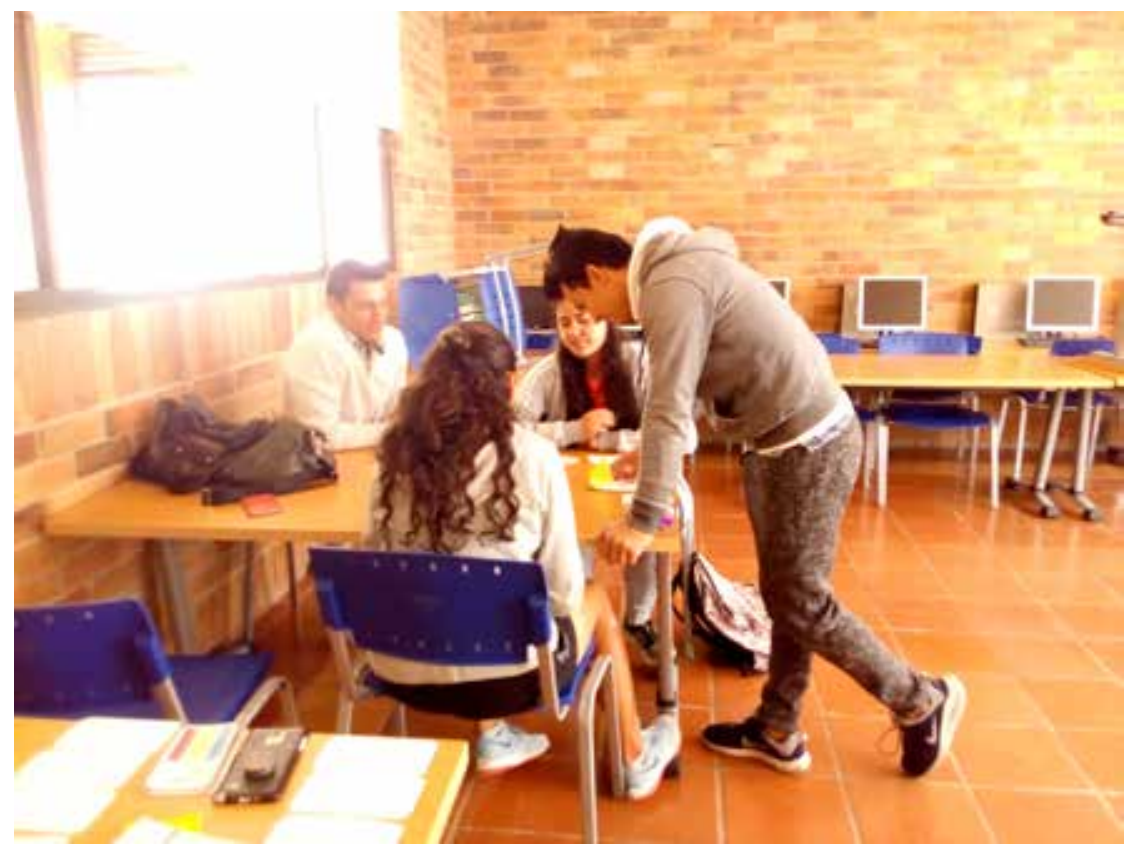


Como se mencionó anteriormente, la investigación pretende evidenciar los aportes de las prácticas recreativas implementadas en la cátedra de Morfofisiología, de esta forma se brindarán alternativas metodológicas que contribuyan al fortalecimiento del proceso enseñanza-aprendizaje por medio de un ambiente lúdico tanto para los estudiantes como para los docentes pertenecientes a la Facultad de Cultura Física, Deporte y Recreación de la Universidad Santo Tomás; en cuanto al ambiente lúdico, Manrique y Gallego (2012) expresan que estos fortalecen en los estudiantes la concentración e inteligencia, estimulan los sentidos y generan esquemas cognitivos más significativos.

Uno de los referentes de mayor importancia que se pretende implementar en las prácticas recreativas es el juego, considerado como un elemento característico y que ha dejado huella en las diversas etapas del desarrollo humano, pues con este se identifica una estrecha relación de conceptos como alegría, disfrute, risa, amigos, compartir, etc. Esto es lo que se quiere lograr con la investigación, generar un espacio en donde, además de que los estudiantes "la pasen bueno", aprendan de manera significativa. A la hora de plantear un juego didáctico se debe tener en cuenta lo expuesto por Chacón (2008), en donde se establece: el objetivo didáctico: el contenido del juego de acuerdo con los conocimientos que se pretenden inculcar; las acciones lúdicas: hacen el proceso más ameno y conquistan la atención de los participantes, y las reglas del juego: dan las pautas para cumplir satisfactoriamente las actividades planteadas.

A través de la historia se ha conocido que "Inicialmente, el currículo de las Escuelas Normales estuvo orientado hacia una pedagogía tradicional, basada en la relación del maestro con los contenidos y después con los métodos” (Tatto y Vélez, 2007, p. 18), por lo tanto, al brindar alternativas metodológicas se puede llegar a solucionar diferentes problemáticas como la falta de interés por la materia, una metodología aburrida y extensa, resultados académicos deficientes, etc. Concordando con lo planteado por López (2011) "los alumnos se aburren a causa de la rutina escolar decepcionante y deplorable, en una escuela tradicional que no les satisface" (p. 17). Estos problemas se pueden relacionar como parte de las consecuencias de una metodología tradicional, pues dentro de sus características se encuentra que la comunicación posee carácter autoritario, de tipo emisor-receptor, vertical, que privilegia la información con énfasis en los productos y no en 
los procesos" (Ortiz, 2008, p. 59), por lo que es importante llegar a una solución oportuna innovando desde el aula para que las falencias se puedan convertir en fortalezas a futuro; dentro de estas oportunidades de mejora se encuentra la recreación, en donde a través de una vivencia planeada, sustentada pedagógicamente y estructurada metodológicamente, se trasciende de la vivencia a una experiencia social enriquecedora (Abella, Fonseca, Piratova y Ruiz, 2018).

En cuanto a la relevancia social y su viabilidad, se estima que al lograr un mejor entendimiento de las temáticas de esta cátedra por parte de los estudiantes y que esos conocimientos sean significativos para ser utilizados posteriormente como base en otras materias, se estará aportando a disminuir el número de estudiantes que pierden la materia y aumentar tanto el promedio general del grupo como el individual, contribuyendo a largo plazo para que los estudiantes terminen su carrera a tiempo, disminuyendo costos educativos y se inicien en el mundo laboral en el momento proyectado. Igualmente, se aspira a que el material didáctico elaborado demuestre la importancia del trabajo en pro de un ambiente lúdico en los espacios institucionales y su impacto en el proceso de enseñanza-aprendizaje de los mismos, para posteriormente ser implementado en las diversas facultades de otras instituciones educativas de nivel superior.

\section{Metodología}

La metodología que se presenta a continuación cuenta con un enfoque mixto, estructurada en dos fases, la fase 1 corresponde al enfoque cuantitativo, mientras que en la fase 2 se desarrolla el enfoque cualitativo.

La población elegida para la ejecución del proyecto se encontró compuesta por todos los estudiantes que cursaban la cátedra de Morfofisiología en el período 2018-1, pertenecientes a la Facultad de Cultura Física, Deporte y Recreación de la Universidad Santo Tomás, Bogotá, sede campus.

La muestra elegida fue de tipo no probabilística, pues los participantes de la investigación fueron sujetos tipo (curso 2D, compuesto por 24 estudiantes) que cumplían con los requerimientos para desarrollar la investigación, estos sujetos habían tenido la experiencia reciente de vivenciar esta cátedra con la docente Angie Grillo y contaban con la representatividad 
teórica para que la muestra fuera válida. Para determinar los efectos que pudiera lograr la metodología en el rendimiento académico de los estudiantes, se decidió dividir la muestra en un grupo control y uno experimental por medio de la asignación aleatoria en un listado de Excel. En la Figura 2, se observa la aplicación de uno de los materiales didácticos denominado twiz-músculo en pequeños grupos, el material se encuentra distribuido en el piso del salón formando una cuadrícula 4 x 4 .

Figura 2. Ejecución del juego twiz-músculo (músculos, manos y pies). Fotografía Juan Pablo Macías

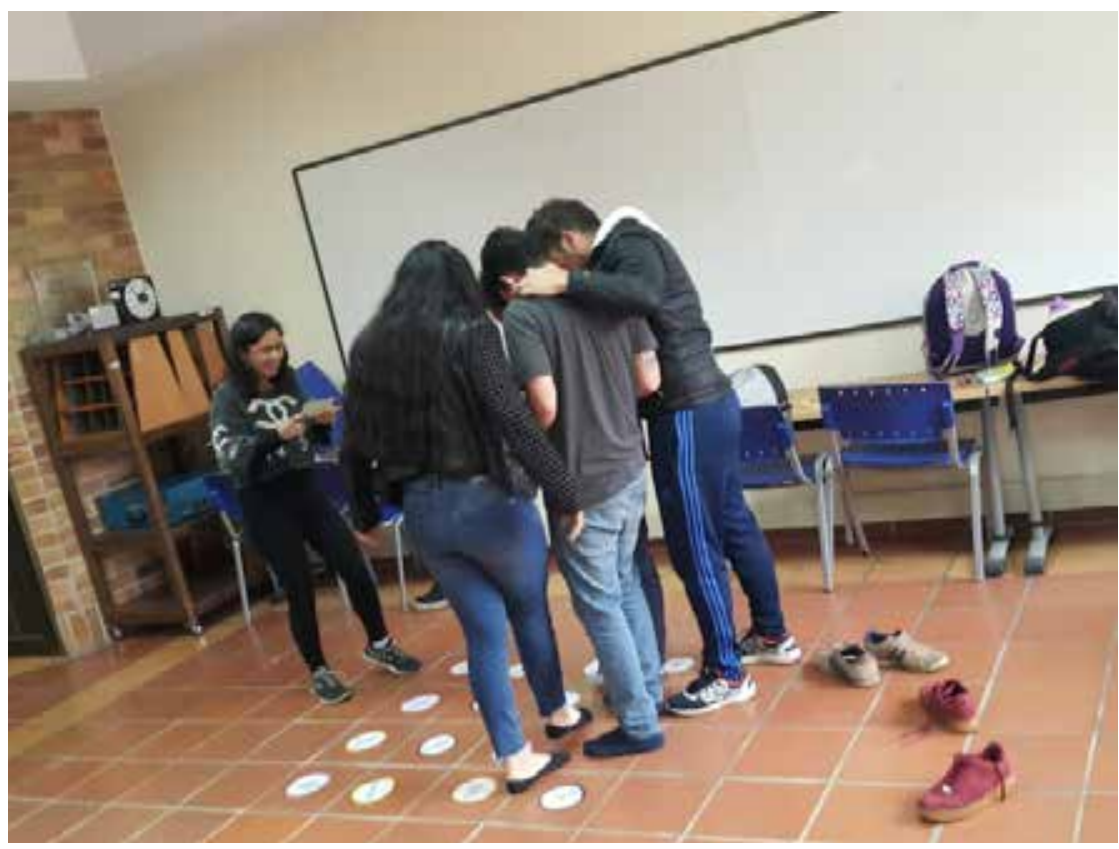

Con el fin de propiciar un ambiente lúdico se realizó la aplicación de tres juegos con el grupo experimental (conformado por 12 estudiantes) en grupos de 4, durante 3 sesiones, en donde se abarcaron las temáticas correspondientes al segundo corte del 2018-1 de la cátedra: origen, inserción y función muscular, a cada uno de estos temas se le asignó un color correspondiente para facilitar el proceso asociativo de los estudiantes. El 
primer juego, denominado "tri-músculo", comprendía una serie de cartas que debían ser ordenadas de tal manera que los aspectos mencionados anteriormente correspondieran a la imagen del músculo, con este juego se abarcaron los segmentos corporales correspondientes a hombro, codo y muñeca. El segundo juego, "twis-músculo", estaba conformado por una serie de fichas circulares que se colocaban formando una cuadrícula en el piso y, de acuerdo con las indicaciones de los facilitadores los participantes debían posicionar determinada parte de su cuerpo sobre el origen, inserción o función de los músculos correspondientes a las manos y pies. El último juego, “¿qué músculo soy?”, un juego de adivinanzas realizado por parejas, consistía en ubicar en la frente de uno de los estudiantes una ficha con los elementos mencionados anteriormente, mientras que su compañero, sin hablar, debía tratar de darle la mayor información posible acerca de la ficha del músculo que tenía ubicada en la frente, aquí se trabajaron los segmentos correspondientes a tronco, rodilla y tobillo, culminando con este los músculos del cuerpo humano.

\section{Fase 1: cuantitativa}

Este estudio fue de tipo explicativo, ya que pretendía determinar el efecto que tiene la variable independiente (prácticas lúdicas) sobre la variable dependiente (tasa de reprobación y rendimiento en el parcial teórico de Morfofisiología, Tabla 1); su diseño fue cuasiexperimental, pues aunque existía comparación entre grupos (control y experimental), la muestra no fue elegida aleatoriamente.

\section{Hipótesis}

- Hi: la tasa de reprobación disminuye como consecuencia de la aplicación de una metodología basada en las prácticas recreativas.

- He: TRe $<\mathrm{TRc}$

$e=$ Grupo experimental

$c=$ Grupo control

- Ho: La tasa de reprobación no disminuye como consecuencia de la aplicación de una metodología basada en las prácticas recreativas. 
Tabla 1. Principales variables dentro de la investigación

\begin{tabular}{|c|c|c|}
\hline Variable & Definición conceptual & Definición operacional \\
\hline $\begin{array}{l}\text { Prácticas } \\
\text { recreativas }\end{array}$ & $\begin{array}{l}\text { Aplicación de nuevos esquemas } \\
\text { que se acomodan a unos ya exis- } \\
\text { tentes permitiendo un aprendiza- } \\
\text { je significativo generado a partir } \\
\text { de experiencias o saberes previos, } \\
\text { llevando a una mayor compren- } \\
\text { sión y asimilación de determi- } \\
\text { nado aprendizaje (Manrique y } \\
\text { Gallego, 2012). }\end{array}$ & $\begin{array}{l}\text { Ausencia o presencia de una } \\
\text { metodología lúdica en la } \\
\text { cátedra. }\end{array}$ \\
\hline $\begin{array}{c}\text { Tasa de } \\
\text { reprobación }\end{array}$ & $\begin{array}{l}\text { El número total de estudiantes } \\
\text { reprobados al final de cada } \\
\text { corte del semestre [(reprobados/ } \\
\text { total estudiantes)*100] (Vidales, } \\
2009) .\end{array}$ & $\begin{array}{l}\text { Resultado del promedio } \\
\text { de estudiantes reprobados } \\
\text { luego de la aplicación de la } \\
\text { fórmula1. }\end{array}$ \\
\hline $\begin{array}{l}\text { Rendimiento } \\
\text { académico }\end{array}$ & $\begin{array}{l}\text { Evaluación del conocimiento ad- } \\
\text { quirido en el ámbito universita- } \\
\text { rio, en la cátedra de Morfofisio- } \\
\text { logía de la Facultad de Cultura } \\
\text { Física, Deporte y Recreación } \\
\text { (Vidales, 2009). }\end{array}$ & $\begin{array}{l}\text { Calificación obtenida luego } \\
\text { del parcial aplicado y desa- } \\
\text { rrollado por el docente eva- } \\
\text { luando los conocimientos } \\
\text { adquiridos de las temáticas } \\
\text { de la cátedra. }\end{array}$ \\
\hline
\end{tabular}

Nota: tomado de variables investigativas. Elaboración propia.

Fuente: elaboración propia. Tomado de variables investigativas.

\section{Resultados y discusión}

Para el análisis estadístico se utilizó la prueba "t student" con el fin de determinar la significancia de las calificaciones entre el grupo control y el grupo experimental. Para poder realizar este análisis estadístico se formularon dos hipótesis:

1. Hi: existe una diferencia significativa entre la media de calificaciones del grupo control y la media de calificaciones del grupo experimental.

2. Ho: no existe una diferencia significativa entre la media de calificaciones del grupo control y la media de calificaciones del grupo experimental. 
Posteriormente a esto se determinó el nivel alfa ( $\alpha$ ), el porcentaje de error que se está dispuesto a correr en la prueba estadística, en este caso del $5 \%$, es decir, 0.05 .

Tabla 2. Resultados de la aplicación de la prueba t-student, tabla de significancia bilateral

\begin{tabular}{ccccccc}
\hline $\mathrm{T}$ & $\mathrm{gl}$ & $\begin{array}{c}\text { Sig. } \\
\text { (bilateral) }\end{array}$ & $\begin{array}{c}\text { Diferencia } \\
\text { de medias }\end{array}$ & $\begin{array}{c}\text { Diferencia } \\
\text { de error } \\
\text { estándar }\end{array}$ & $\begin{array}{c}95 \% \text { de intervalo } \\
\text { de confianza de la } \\
\text { diferencia }\end{array}$ \\
\hline & & & & & Inferior & Superior \\
0.699 & 22 & 0.492 & 1.2667 & 1.8124 & -2.4920 & 5.0253 \\
0.699 & 19.238 & 0.492 & 1.2667 & 1.8124 & -2.5236 & 5.0569 \\
\hline
\end{tabular}

Fuente: elaboración propia. Tomado de significancia bilateral t-student.

Como se puede observar en la Tabla 2, la significancia (bilateral) es superior al valor de P-valor $=0.05$, por ende, se interpreta que las calificaciones del grupo control y las calificaciones del grupo experimental no tienen un alto grado de significancia. Por lo tanto no se rechaza la hipótesis nula y se acepta.

\section{Fase 2: cualitativa}

Este estudio fue de tipo microetnográfico debido a que se pretendía obtener datos de las experiencias de un grupo reducido de personas y por medio de estos intentar producir un cambio en el estilo de aprendizaje de los estudiantes. A continuación se observan en la Figura 3 las categorías establecidas antes y durante el desarrollo del proyecto, en la cual se explica la correspondencia de colores que allí se muestra. 
Figura 3. Árbol de categorías. En color azul se puede observar el objeto de estudio de la investigación, seguido de la segmentación poblacional. En amarillo se encuentran las categorías a priori y en naranja las categorías emergentes con sus códigos respectivamente

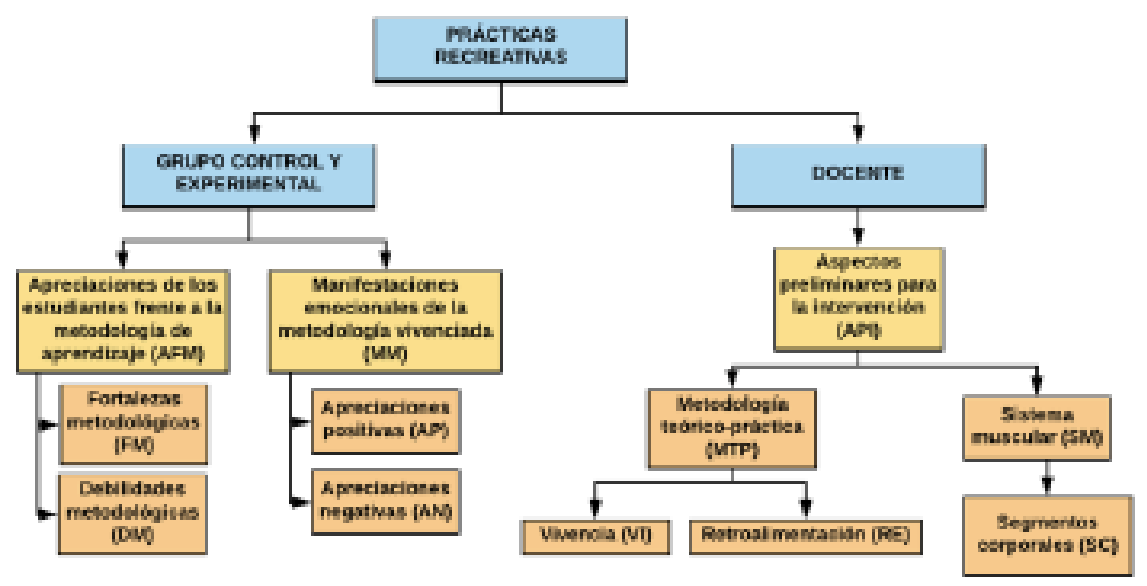

Fuente: elaboración propia.

Estrategias y técnicas de recolección de datos: la estrategia que orientó este estudio fue la narración y la conversación, en cuanto a las técnicas de recolección de datos se aplicó un cuestionario a la muestra poblacional que se elaboró con base a las categorías a priori de las apreciaciones de los estudiantes frente a la metodología de aprendizaje (AFM) y las manifestaciones emocionales de la metodología evidenciada (MM), al igual que una entrevista casual a la docente encargada de la cátedra con el fin de establecer los aspectos relacionados con la categoría aspectos preliminares para la intervención (API).

En la Tabla 3 que se presenta a continuación, se observa parte de la organización de la información recolectada en las matrices finales, teniendo en cuenta las categorías mencionadas anteriormente y las intervenciones realizadas con el grupo control y experimental. 


\section{Resultados}

\section{Tabla 3. Matriz comparativa e interpretativa grupo control $v s$. grupo experimental}

\begin{tabular}{|c|c|c|c|}
\hline \multicolumn{4}{|c|}{ Comparacion del grupo control con el experimental } \\
\hline AFM & & MM & \\
\hline Convergencias & Divergencias & Convergencias & Divergencias \\
\hline $\begin{array}{l}\text { A nivel general el grupo co- } \\
\text { menta que los aspectos po- } \\
\text { sitivos de la metodología es } \\
\text { que es dinámica, permite vi- } \\
\text { venciar los conceptos y que } \\
\text { es atractiva. En cuanto a } \\
\text { aspectos negativos están de } \\
\text { acuerdo de que el tiempo en } \\
\text { el cual se aplican estas dos } \\
\text { metodologías es muy corto, } \\
\text { lo que no permite que las } \\
\text { actividades sean tan fructi- } \\
\text { feras como se espera. }\end{array}$ & $\begin{array}{l}\text { Hay varias divergencias sig- } \\
\text { nificativas, la primera de estas } \\
\text { es debido a la densidad de con- } \\
\text { ceptos la cual es mencionada } \\
\text { en el grupo control y no en el } \\
\text { experimental, se puede deter- } \\
\text { minar que puede ser debido a } \\
\text { la manera como se transmiten } \\
\text { los términos, por un lado una } \\
\text { metodología dinámica, que } \\
\text { vivencia conceptos y por otro } \\
\text { lado una metología recreativa } \\
\text { que la definen como motivan- } \\
\text { te, atractiva, etc. Por otro lado } \\
\text { se encuentran divergencias en } \\
\text { cuanto a la descripción de la } \\
\text { metodología, lo que quiere de- } \\
\text { cir que el grupo control define } \\
\text { esta metodología con los dos } \\
\text { conceptos más usados por los } \\
\text { alumnos como lo son metodo- } \\
\text { logías dinámica y experiencial, } \\
\text { por otro lado el grupo experi- } \\
\text { mental define la metodología } \\
\text { lúdica con gran variedad de } \\
\text { conceptos pero entre esos los } \\
\text { más usados son: lúdica, moti- } \\
\text { vacional, divertida, ayuda a re- } \\
\text { forzar conceptos e interactiva. }\end{array}$ & $\begin{array}{l}\text { A nivel general las ma-nifes- } \\
\text { taciones del grupo son bue- } \\
\text { nas pero hay una convergen- } \\
\text { cia negativa para las dos y es } \\
\text { el hecho de que no todas las } \\
\text { veces el alumno está ubicado } \\
\text { dentro de las actividades, lo } \\
\text { que quiere decir que hace } \\
\text { falta un poco más de intro- } \\
\text { ducción en la aplicación de } \\
\text { las prácticas o de la clase } \\
\text { como tal. }\end{array}$ & $\begin{array}{l}\text { En este aspecto las mani- } \\
\text { festaciones se centran en } \\
\text { que son buenas, pero hay } \\
\text { una gran diferencia en la } \\
\text { que ellos describen esas } \\
\text { manifestaciones, en el gru- } \\
\text { po control la manifesta- } \\
\text { ción es buena debido a lo } \\
\text { dinámica y fructífera que } \\
\text { es la metodología, además } \\
\text { mencionan que en cuanto } \\
\text { a aspectos negativos en } \\
\text { ocasiones sienten algo de } \\
\text { impotencia y estrés, por } \\
\text { otro lado el grupo experi- } \\
\text { mental menciona que esta } \\
\text { manifestación se puede lle- } \\
\text { gar a describir gracias a la } \\
\text { sociabilidad y asociaciones } \\
\text { que pueden lograr gracias } \\
\text { a las actividades, lo cual } \\
\text { las encasillan en algo más } \\
\text { profundo y importante a } \\
\text { la hora de transmitir un } \\
\text { concepto. }\end{array}$ \\
\hline
\end{tabular}


Matriz interpretativa

\begin{tabular}{|c|c|c|c|}
\hline \multicolumn{2}{|l|}{$\mathrm{AFM}$} & \multirow{2}{*}{$\frac{\text { MM }}{\text { Control }}$} & \\
\hline Control & Experimental & & Experimental \\
\hline $\begin{array}{l}\text { El control define la meto- } \\
\text { dología como buena, debi- } \\
\text { do a que es descrita por los } \\
\text { estudiantes como dinámica, } \\
\text { experiencial y atractiva. } \\
\text { Por otro se menciona una } \\
\text { divergencia entre el roll que } \\
\text { cumple el docente dentro } \\
\text { del aula, en la cual hay una } \\
\text { división de opiniones, unas } \\
\text { que lo describen como una } \\
\text { buena participación y otra } \\
\text { como falta de interacción } \\
\text { con las prácticas, para fi- } \\
\text { nalizar debe mejorar varios } \\
\text { aspectos los cuales los men- } \\
\text { cionan los alumnos tales } \\
\text { como: el tiempo en que se } \\
\text { realizan las actividades, la } \\
\text { densidad de los conceptos } \\
\text { a trabajar y el gran proceso } \\
\text { de memorización que con- } \\
\text { lleva la materia. }\end{array}$ & $\begin{array}{l}\text { El experimental es un poco } \\
\text { más profundo al definir la } \\
\text { apreciación con respecto a la } \\
\text { metodología, en general es } \\
\text { mencionada como buena pero } \\
\text { hay una gran variedad de con- } \\
\text { ceptos mencionados en las res- } \\
\text { puestas que dan a entender el } \\
\text { impacto que tuvieron las prác- } \\
\text { ticas en este grupo, los concep- } \\
\text { tos más usados para definir la } \\
\text { metodología fueron: divertida, } \\
\text { lúdica, motivante, novedosa, } \\
\text { interactiva, social, detallada, } \\
\text { clara y completa. del mismo } \\
\text { modo hubo varias respuestas } \\
\text { que mencionaron que esta } \\
\text { metodología realizaba asocia- } \\
\text { ciones con gran facilidad. Por } \\
\text { otro lado también presenta } \\
\text { falencias tales como la falta de } \\
\text { tiempo y la no retroalimenta- } \\
\text { ción al final. }\end{array}$ & $\begin{array}{l}\text { El control define sus relación } \\
\text { con la actividad como bue- } \\
\text { na por la gran mayoría de } \\
\text { los alumnos, debe ser por la } \\
\text { buena interacción que tienen } \\
\text { con esta como se menciona- } \\
\text { ba en la categoría AFM, aún } \\
\text { hay manifestaciones que se } \\
\text { deben procurar mejorar ta- } \\
\text { les como la impotencia, el } \\
\text { estrés y la falta de ubicación } \\
\text { del alumno, lo que quiere } \\
\text { decir que ellos no están ne- } \\
\text { tamente metidos dentro del } \\
\text { tema lo que hace que no in- } \\
\text { teractúen en las actividades } \\
\text { como debe ser. }\end{array}$ & $\begin{array}{l}\text { La manifestación del } \\
\text { grupo experimental con } \\
\text { respecto a la metodología } \\
\text { concuerdan en que es bue- } \\
\text { na debido al aspecto social, } \\
\text { eficiente y la capacidad de } \\
\text { asociación que genera la } \\
\text { metodología, aun así hay } \\
\text { un aspecto a tener en cuen- } \\
\text { ta y es la ubicación inicial } \\
\text { de los alumnos, esto quiere } \\
\text { decir que se debe asegurar } \\
\text { al inicio de las prácticas de } \\
\text { que estos entendieron la } \\
\text { actividad de manera com- } \\
\text { pleta y se sien-ten a gusto } \\
\text { con su grupo de trabajo. }\end{array}$ \\
\hline
\end{tabular}

A continuación se presentan las conclusiones identificadas en la matriz interpretativa de acuerdo con las categorías definidas anteriormente: apreciaciones de los estudiantes frente a la metodología de aprendizaje (AFM) y manifestaciones emocionales de la metodología vivenciada (MM), expresadas tanto por el grupo control como por el experimental.

\section{Grupo control:}

- Los estudiantes definen la metodología de la docente como dinámica, experiencial y atractiva. En la Figura 4 se observa una de las estrategias que la docente implementa en su clase, basada en el reconocimiento físico de algunos huesos y la identificación de los accidentes óseos por medio de la ubicación de papelitos que llevan los distintos nombres de los mismos.

- Los aspectos que se deben mejorar en la metodología de acuerdo con las opiniones de los estudiantes son: el tiempo en el que se realizan las actividades, la densidad de los conceptos a trabajar y el proceso de memorización que implica la materia. 
- Emocionalmente los estudiantes describen la metodología como buena, aunque también se identificó que durante la clase se presentan casos de estrés, impotencia y falta de ubicación del alumno con las temáticas.

Figura 4. Una de las estrategias que utiliza la docente en su clase (accidentes óseos en cada hueso). Fotografía Melisa Narváez, 2018.

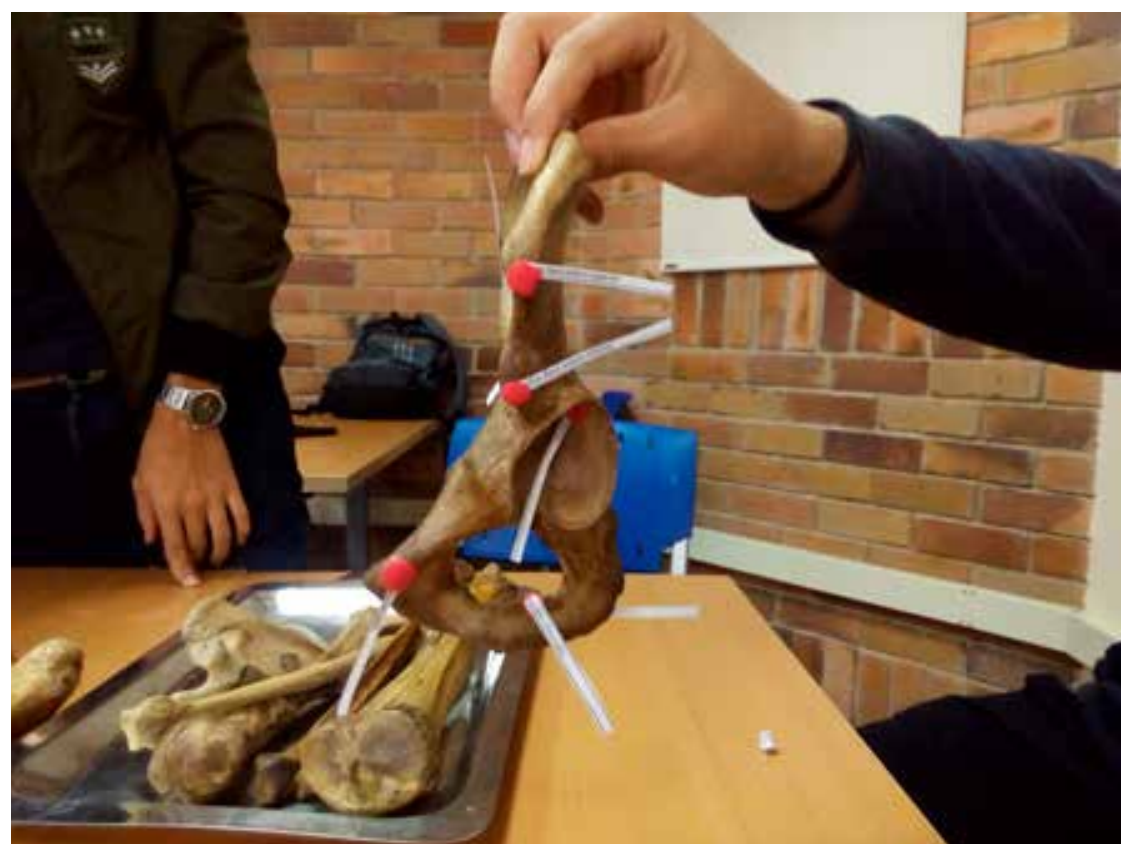

\section{Grupo experimental:}

- Los estudiantes describen la metodología alternativa como buena, en donde se resaltan algunos conceptos expresados por ellos relacionados con felicidad, tales como lúdica, motivante, novedosa y completa; en aspectos por mejorar se resalta la falta de tiempo y la falta de una retroalimentación final. En la Figura 5 se observa el desarrollo del juego tri-músculo en uno de los grupos, los estudiantes se encuentran en la fase de reconocimiento de las fichas y la organización de las mismas 
logrando que concuerde tanto la imagen del músculo junto con su origen, inserción y función.

- Frente a los aspectos emocionales los estudiantes manifiestan que la metodología tuvo un impacto positivo en el grupo, resaltando aspectos como cohesión grupal, el fomento del aprendizaje por medio de asociaciones y la eficiencia del método en la medida en que ellos recuerdan conocimientos clave desarrollados en los juegos, pues según estos, Melo y Hernández (2014) exponen que "refuerza y agudiza determinada capacidad física o intelectual, por el camino del placer o de la obstinación, hace fácil lo que en un principio fue difícil o agotador" (p. 43). En aspectos por mejorar mencionan la claridad a la hora de dar las instrucciones del juego y asegurarse que exista un ambiente cómodo en el grupo de trabajo.

Figura 5. Desarrollo del juego "tri-músculo" (músculos hombro, codo y muñeca). Fotografía Juan Pablo Macías, 2018

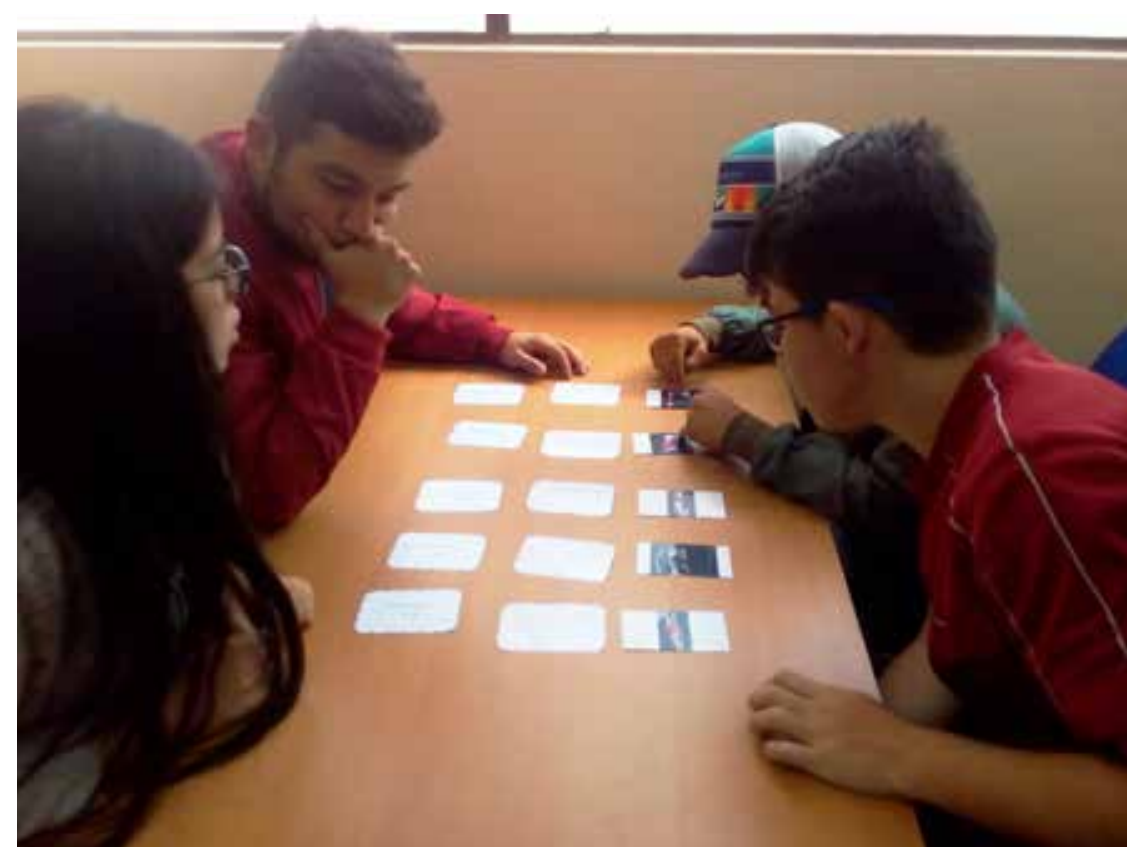




\section{Conclusiones}

La estrategia de implementar prácticas lúdicas en materias que son teóricamente muy densas resulta ser una herramienta importante para la transmisión de conocimiento, siendo uno de los objetivos implícitos de esta metodología tratar de trascender la forma de aprendizaje a uno significativo, lo cual permite relacionar los conocimientos adquiridos con los esquemas cognitivos estructurados anteriormente.

Se reconoce que las materias que están fuera del módulo de recreación como lo es Morfofisiología pueden fortalecer su metodología por medio de la recreación, ya que esta al ser entendida como un campo, permite brindar estrategias y soluciones a distintas problemáticas.

La investigación presenta una relevancia importante para el futuro de las metodologías de enseñanza, así pues, se deja una propuesta tentativa de mezclar una metodología tradicional con una metodología lúdica, de esta manera puede ser más factible la transmisión de conocimientos y el aumento del rendimiento académico por parte de los estudiantes.

Es importante resaltar que no se debe dejar completamente de lado la metodología tradicional, ya que aporta bases y es necesaria en la creación de esquemas mentales en los estudiantes, los cuales ayudan a comprender e interiorizar mejor la información. En lo que se puede trabajar es en la modificación de algunas de las características que ha representado esta metodología a lo largo de la historia, como el carácter autoritario y el énfasis en un resultado exitoso.

\section{Referencias}

Abella, M., Fonseca, F., Piratova, D. y Ruiz, L. (2018). Módulo de profundización en recreación y manejo de tiempo. Universidad Santo Tomás, Bogotá D. C., Colombia. Recuperado de http://repository.usta.edu.co/handle/11634/10592

Anzola, C., Castellanos, M., Orduz, D. Salazar, I. y Sánz, A. (2013). Creatividad, lúdica y juego: opciones transformadoras en contextos educativos con adultos. Universidad de San Buenaventura: Bogotá D. C., Colombia. 
Calzadilla, R. (2004). La pedagogía como ciencia humanista: conocimiento de síntesis, complejidad y pluridisciplinariedad. Revista de Pedagogía, 25(72), 123148. Recuperado de http://www.scielo.org.ve/scielo.php?script=sci_arttext\&pi $\mathrm{d}=$ S0798-97922004000100005

Chacón, P. (2008). El juego didáctico como estrategia de enseñanza y aprendizaje. ¿Cómo crearlo en el aula? Nueva Aula, 16(5). DOI: https://doi.org/10.14201/ aula201723303318.

López, N. (2011). Apuntes sobre la pedagogía crítica. Bogotá, Colombia. Editorial B - Universidad Santiago de Cali. Recuperado de https://issuu.com/bibliotecaupn/ docs $/ 13 \mathrm{f}$

Manrique, A. y Gallego, A. (2012). El material didáctico para la construcción de aprendizajes significativos. Revista Colombiana de Ciencias Sociales, 4(1), 101-108. Recuperado de https://www.redalyc.org/pdf/4978/497856284008. pdf

Melo, M. y Hernández, R. (2014). El juego y sus posibilidades en la enseñanza de las ciencias naturales. Innovación Educativa, 14(66), 41-63. Recuperado de http:// www.scielo.org.mx/pdf/ie/v14n66/v14n66a4.pdf

Ortiz, T. (2008). Comunicarse y aprender en el aula universitaria. La Habana, Cuba: Editorial Universitaria. Recuperado de https://www.google.com/url?sa =t\&source=web\&rct=j\&url=http://beduniv.reduniv.edu.cu/fetch.php\%3Fda ta \%3D196\%26type\%3Dpdf\%26id\%3D2809\%26db\%3D0\&ved=2ahUKE widxL7S7KziAhXFjFkKHRgqAXcQFjAAegQIARAB\&usg=AOvVaw085gh 8X_xvN9mu1xEQ4T7T

Posada, R. (2014). La lúdica como estrategia didáctica. (tesis para optar por el título de magister en Educación con Énfasis en Ciencias de la Salud). Universidad Nacional de Colombia, Bogotá D. C., Colombia. DOI: https://doi. org/10.15381/os.v8i2.3141

Rodríguez, M. (2004). La teoría del aprendizaje significativo. Santa Cruz de Tenerife, España: Centro de Educación a Distancia - CEAD.

Sarlé, M. (2001). Juego y aprendizaje escolar. Los rasgos del juego en la educación infantil. Buenos Aires, Argentina: Editorial Novedades Educativas.

Tatto, M. y Vélez, E. (2007). Iniciativas para el cambio en la formación de maestros: el caso de México. Revista Latinoamericana de Estudios Educativos, 29 (3-4), 9-62. DOI: https://doi.org/10.18172/con.2837

Torres, H. y Girón, D. (2009). Didáctica general. San José, Costa Rica: Editorama S. A. 
Vidales, S. (2009). El fracaso escolar en la educación media superior. El caso del bachillerato de una universidad mexicana. REICE: Revista Electrónica Iberoamericana sobre Calidad, Eficacia y Cambio en Educación,7 (4), 321341. DOI: https://doi.org/10.25074/07195532.16.300

Zuluaga, C. y Gómez, M. (2016). Metodología lúdica para la programación dinámica determinista en un contexto universitario. Entramado, 12(1), 236-249. DOI: https://doi.org/10.18041/entramado.2016v12n1.23124 